Check for updates

Cite this: RSC Adv., 2017, 7, 52930

Received 11th October 2017

Accepted 2nd November 2017

DOI: $10.1039 / c 7 r a 11191 f$

rsc.li/rsc-advances

\section{High performance transient organic solar cells on biodegradable polyvinyl alcohol composite substrates $\uparrow$}

\author{
He Xi, ID $t^{* a}$ Dazheng Chen, $t^{b}$ Ling LV, ${ }^{a}$ Peng Zhong, ${ }^{a}$ Zhenhua Lin, ${ }^{b}$ \\ Jingjing Chang, $\mathbb{D}^{\mathrm{b}}$ Hong Wang, ${ }^{a}$ Bin Wang, ${ }^{\mathrm{ac}}$ Xiaohua Ma*a and Chunfu Zhang ${ }^{*}{ }^{* b}$
}

Physically transient electronics represent an emerging class of technology which can disappear in a controlled manner when triggered by stimuli. Transient power supply devices are essential components in meeting the power demands of transient systems. Here we report on the first demonstration of transient organic solar cells (OSCS) fabricated on polyvinyl alcohol (PVA) composite substrates. PVA is employed as a substrate material because of its high transparency, good water solubility and versatility in chemical and physical properties by appropriate choice of composition. The dissolution and transparency of PVA substrates can be programmed by addition of sucrose or gelatin at different ratios, which further define the photovoltaic performance and transiency behavior of the transient OSCs. Based on the poly(3-hexylthiophene):[6,6]-phenyl- $\mathrm{C}_{61}$-butyric acid methyl ester (P3HT:PC ${ }_{61} \mathrm{BM}$ ) system, a power conversion efficiency up to $2.05 \%$ is obtained for OSCs fabricated on a PVA/sucrose substrate with a ratio of $2: 1$ (PS2), which is comparable to that of the reference devices on glass substrates (2.37\%). Once triggered by deionized water, the devices disconnect within only $10 \mathrm{~s}$ exposure to stimuli. When using a PVA/gelatin composite with a composition of $2: 1$ (PG2) as substrate, the disintegration is significantly prolonged. The devices can maintain their integrity even after an hour of exposure, however, in forms of individual particles. Efficient and easy fabrication of transient OSCs on the PVA-based substrates is expected to open the door for transient organic photovoltaic technology.

\section{Introduction}

Physically transient electronic systems that are designed to undergo disintegration in a controlled manner with minimal or untraceable remains are of great interest for biomedical implants, hardware-secure electronics, disposal consumer electronics, degradable remote environmental monitors/sensors, and other devices. ${ }^{1-8}$ The constituent materials used in transient systems play a critical role in defining the transient process and transience time for such systems. ${ }^{9}$ Despite the degradable

\footnotetext{
${ }^{a}$ School of Advanced Materials and Nanotechnology, State Key Laboratory of Wide Band Gap Semiconductor Technology, Xidian University, 266 Xinglong Section of Xifeng Road, Xi'an, 710126, China.E-mail: hxi@xidian.edu.cn; xhma@xidian.edu.cn ${ }^{b}$ State Key Laboratory of Wide Band Gap Semiconductor Technology, School of Microelectronics, Xidian University, 2 South Taibai Road, Xi'an, 710071, China. E-mail: cfzhang@xidian.edu.cn

'Key Laboratory of Microelectronic Devices \& Integrated Technology, Institute of Microelectronics, Chinese Academy of Sciences, Beijing, 100029, China

$\dagger$ Electronic supplementary information (ESI) available: Denotations for each sample; Optical and AFM images of PVA composites thin films; Schematic diagram of fabrication process for PVA substrates and transient OSCs; IPCE spectra of transient OSCs; Pictures illustrating time sequence of the dissolution of transient OSCs. See DOI: 10.1039/c7ra11191f

$\ddagger$ Dr H. Xi and D. Chen contribute equally on this work.
}

properties, these components need to offer full characteristics and reliable, high-performance operation in normal use. Recent advanced transient systems have exploited biocompatible inorganic/organic electronic materials such as monocrystalline silicon nanomembranes, ${ }^{1,10,11}$ silicon-germanium, ${ }^{12}$ or metal oxide (e.g. zinc oxide, indium-gallium-zinc-oxide) ${ }^{3,13}$ as semiconductors, magnesium oxide, ${ }^{4,14}$ silicon oxides or nitrides ${ }^{15,16}$ for dielectrics, with metals including $\mathrm{Mg}$, Zn, Mo, $\mathrm{W}$, and Fe for the electrodes and interconnects., ${ }^{6,17,18}$ Additionally, organic polymeric materials, which are typically biocompatible, biodegradable and convenient to fabricate, have been utilized as substrates and encapsulation layers for transience applications. Demonstrated transient devices built with biodegradable polymers include field effect transistors, zero-waste strain/ temperature/hydration sensors, diodes, and complementary metal-oxide-semiconductor ring oscillators. ${ }^{1-7,11-15,19-23}$ Previous work establishes options that include films of poly(vinyl alcohol) (PVA), poly(L-lactic-co-glycolide) (PLGA), poly(lactic acid) (PLA), polycaprolactone (PCL), rice paper, cellulose, and silk fibroin. In most cases, the life cycle of the device and timescale of transience is dominated by the dissolution rate of polymer substrates and packaging layers, which can be tuned by film crystallinity, thickness, and the use of additives. ${ }^{9,24,25}$ This also means the 
biodegradable polymers are particularly applicable to design and accommodate a programmed transiency process.

For these transience applications, it is highly desirable to develop transient power supply devices to fulfil the power demands of such systems, enabling their reliable operation by supplying sufficient electric energy. On the other hand, the transient design in power supply may provide a viable way for the future management of the continuous growing of energy sucrose waste, which is mainly due to the extensive use of electric vehicles and portable electronics. Reported power supply strategies range from silicon solar cells, mechanical energy harvesters, to external radio frequency power transfer modules and batteries. ${ }^{3,4,7,26-29}$ As it converts solar energy, the largest renewable source, to electric power, photovoltaic devices especially organic solar cells (OSCs) have showed great advantage over other power supply strategies because of their potential for low-cost fabrication, light weight, good mechanical flexibility, and compatibility with various classes of substrates such as plastic, glass, paper and cellulose nanomaterials. ${ }^{30-32}$ Recently, the power conversion efficiency (PCE) of OSCs based on bulk heterojunctions of a donor-acceptor blend has been improved up to values over $10-12 \%{ }^{33}$ As to this meaning, the design of transient organic photovoltaic devices is highly desirable, opening the door for the future development of transient photovoltaic technology.

In this contribution, as a proof-of-concept, we employ PVA composites as the transparent transient substrates and report the physically transient organic solar cells based on poly(3hexylthiophene):[6,6]-phenyl- $\mathrm{C}_{61}$-butyric acid methyl ester $\left(\mathrm{P} 3 \mathrm{HT} / \mathrm{PC}_{61} \mathrm{BM}\right)$ blends for the first time. PVA, which is insulated and can be processed in water at a low temperature, is utilized as the substrate due to its desirable optical transmittance, flexibility, mechanically robust and high water-solubility. ${ }^{34}$ Importantly, the dissolution and transiency of PVA films can be programmed by introduction of fillers to the PVA polymer matrix. Also, the ability of PVA to be served as substrates and encapsulation layers for various transient electronic devices has been demonstrated..$^{35,36}$ In this study, we used sucrose and gelatin as fillers to functionalize PVA matrices, aiming to construct transient polymeric substrates with controllable degradation rate. Sucrose was selected to enhance the dissolution, and as a cross-linker to provide inter-chain interactions, thus improving the mechanical properties of the polymer. ${ }^{37}$ Gelatin is a protein polymer obtained by partly denaturalization of collagen and used to adjust the transiency rate of the polymer composites. The PVA/gelatin blend films can be tuned from soluble to insoluble via varying the ratio of gelatin in the blends, which is ascribed to the triple-helix structure in gelatin. ${ }^{38,39}$ Based on these PVA/sucrose and PVA/gelatin substrates, transient OSCs were fabricated with the configuration of PVA-based substrates $/ \mathrm{Ag} / \mathrm{MoO}_{3} / \mathrm{P} 3 \mathrm{HT}: \mathrm{PC}_{61} \mathrm{BM} / \mathrm{Ca} / \mathrm{Ag}$. The effects of PVA composites with different ratios of sucrose and gelatin on properties of the substrates, OSC device performance and the corresponding transiency behaviour has been investigated. It was found that the dissolution and transparency properties of the transient substrates can be enhanced or reduced by appropriate choice of the compositions. A highest power conversion efficiency (PCE) of $2.05 \%$ is achieved for the devices fabricated on PVA/sucrose substrate with ratio of $2: 1$ (PS2). Interestingly, because of the very fast expansion of PS2 substrates, the devices disintegrate in only $10 \mathrm{~s}$ exposure to deionized (DI) water. The transiency time is much shorter than devices on PVA (20 s) and PVA/gelatin (80 s, PVA/gelatin $=2: 1)$ substrates, suggesting that the strong dependence of overall transiency behavior of the transient solar cells on the that of the substrates. The successful fabrication of transient OSCs on PVAbased substrates would provide valuable guidelines for future development in transient self-powered systems.

\section{Results and discussion}

As the properties of polymer films are closely associated with the structure of composites, Fourier transform infrared (FTIR) spectra were measured to gain insight into the interactions between PVA and fillers. Fig. 1 shows the FTIR spectra of PVA/ sucrose and PVA/gelatin composites. The corresponding denotations for each sample are presented in Table S1, $\uparrow$ the samples with PVA-to-sucrose (or gelatin) weight ratios of $10: 1,2: 1$, $1: 1,1: 2$ are notated as PS10 (PG10), PS2 (PG2), PS1 (PG1) and PSO.5, respectively. The broad bands around $3313-3270 \mathrm{~cm}^{-1}$ for all polymer films assign to the stretching vibration of hydroxyl groups originated from the intermolecular and intramolecular hydrogen-bond interactions, and the intensity of the bands depends on the degree of the hydrogen bonded bands. ${ }^{24,40}$ The double bands around $2910-2937 \mathrm{~cm}^{-1}$ concern the alkyl stretching vibration of PVA, which are not affected by the sucrose and gelatin. Band for $\mathrm{C}-\mathrm{O}$ stretching vibration of secondary alcoholic groups is observed at $1085 \mathrm{~cm}^{-1}{ }^{38}$ The symmetric C-C stretching vibration of PVA is observed at $1141 \mathrm{~cm}^{-1}$, which is the characteristic of semi-crystalline PVA. As can be seen in Fig. 1a, this band becomes more pronounced with increasing sucrose content, indicating the formation of more crystalline domains in polymers. This is primarily caused by the chemical interactions between sucrose and PVA chains. It has been found that the dissolution rate of PVA polymers in water is proportional to the degree of crystallinity, thus fast dissolution of PVA/sucrose blends can be expected, especially when the sucrose content is equal or higher than that of PVA. ${ }^{37}$ The FTIR spectra of PVA/sucrose polymers also show absorption bands at $1416 \mathrm{~cm}^{-1}, 1329 \mathrm{~cm}^{-1}$ and $990 \mathrm{~cm}^{-1}$ due to the bending vibrations of $\mathrm{C}-\mathrm{O}-\mathrm{H}$ groups of sucrose, $\mathrm{C}-\mathrm{O}$ stretching
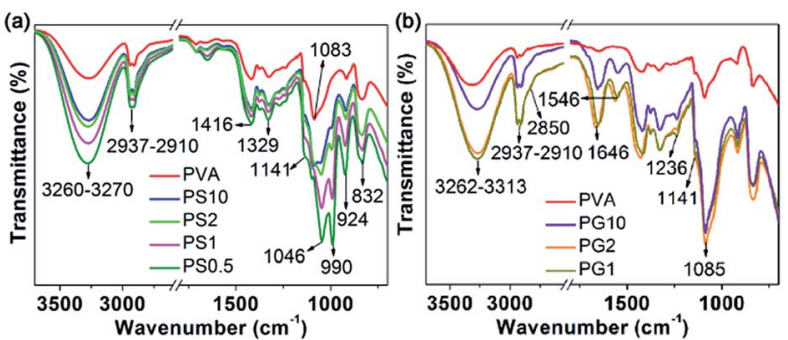

Fig. 1 ATR-FTIR spectra of PVA/sucrose and PVA/gelatin substrates with varying composition. 
vibrations at $1046 \mathrm{~cm}^{-1}$ and $\mathrm{C}-\mathrm{C}$ stretching vibrations at $924 \mathrm{~cm}^{-1}$ and $832 \mathrm{~cm}^{-1}$.

The characteristic absorption bands of gelatin mainly appear around $1655 \mathrm{~cm}^{-1}$ (amide $\mathrm{I}, \mathrm{C}=\mathrm{O}$ and $\mathrm{C}-\mathrm{N}$ stretching vibrations), $1550 \mathrm{~cm}^{-1}$ (amide $\mathrm{II}, \mathrm{N}-\mathrm{H}$ bending vibration) and $1264 \mathrm{~cm}^{-1}$ (amide III, C-N stretching vibration), and all of them are sensitive to the conformational change of gelatin. It can be obviously seen from Fig. 1b that these absorption bands have shifted to lower wavenumbers at 1646, 1546 and $1236 \mathrm{~cm}^{-1}$, respectively, indicating the esterification reaction between PVA and gelatin. ${ }^{\mathbf{4 1}}$ The shoulder band identified as the $\mathrm{C}-\mathrm{H}$ stretching of aldehyde groups of gelatin is showed at $2850 \mathrm{~cm}^{-1}$. The FTIR results suggest good compatibility and chemical interactions between PVA matrix and the fillers, which could substantially determine the chemical and physical properties of the PVA polymer substrates.

For transient applications, the substrates that are dissolvable and have controllable dissolution rate are critically important for such systems. In our study, the PVA composites are very stable in ambient condition for months. Once transiency is triggered by exposure to water, these polymeric substrates undergo fast degradation. The time-dependence dissolution of PVA/sucrose and PVA/gelatin substrates with varying composition were measured by recording the weight loss of the substrates, as presented in Fig. 2. The dissolution rate and solubility varies with the PVA-to-filler ratios. The PVA/sucrose substrates manifest rapid dissolution in the first few minutes of triggering. Their dissolution is highly dependent on the sucrose concentration (Fig. 2a). When low concentrations of sucrose were added, the dissolution of substrates is slightly reduced because of the chemical interactions between sucrose and PVA. ${ }^{24}$ However, increasing the sucrose ratio to equal or higher than PVA leads to much faster dissolution of the substrates than that of PVA, which is probably due to the relatively higher water solubility of sucrose than PVA. Moreover, with the increase of sucrose concentration, more crystal domains were formed due to the increasing numbers of interaction between sucrose and PVA chains, the increased crystallinity in turn contributed to the fast dissolution of sucrose-dominated substrates (PS1 and PS0.5) as discussed above. While for PVA/gelatin substrates, both the dissolution rate and solubility are greatly reduced with increasing amount of gelatin (Fig. 2b). The dissolution is mainly affected by the cross-linking degree within in the composites, which to some
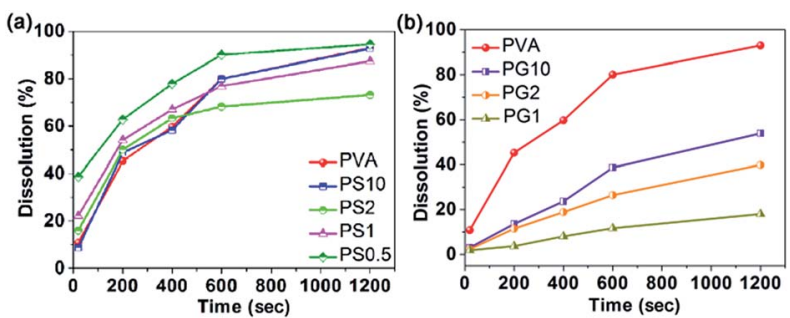

Fig. 2 Plots of dissolution for PVA/sucrose (a) and PVA/gelatin (b) composites as a function of the exposure time in DI water. extent is related to the esterification reaction of the hydroxyl group of PVA with the carboxyl group of gelatin. ${ }^{38}$ The esterification reaction numbers are increased with high gelatin concentrations. The ester bonds, however, especially the carbonyl oxygen atoms are usually used as hydrogen bond acceptors rather than donors, thus essentially decreasing the water solubility of PVA/gelatin substrates. This is well consistent with the observation in FTIR analysis.

In order to evaluate the capability of these polymeric films to serve as the OSCs substrates, the transmittance spectra of the free-standing PVA composite films with varying compositions was measured by UV-vis spectroscopy. The transmittance of glass substrate was also examined for reference. As can be seen in Fig. 3, PVA films have good transmittance in the visible wavelength range with transparency above 91\%, which is comparable to the glass substrate ( 93\%). Addition of sucrose has little effect on the transparency of PVA films (Fig. 3a and S1 in the ESI $\dagger$ ). All the PVA/sucrose films exhibit high transmittance of above $89 \%$, enabling sufficient incident sunlight to pass through the substrate. Among all sucrose-based substrates, the optical transparency of sucrose-dominated films is slightly lower than that of other films. The decrease in transmittance is probably due to the scattering, induced by the random distribution of crystalline domains in PVA/sucrose films. As for gelatin-based polymer films (Fig. 3b), increasing the gelatin content greatly decreased the transmittance of the films from $\sim 88 \%$ (PG10) to $\sim 22 \%$ (PG1), which can be attributed to the interpenetrating networks (IPN) in PVA/ gelatin composites. With increasing amount of gelatin in polymer, more gelatin reacts with PVA and leads to higher crosslinking density, thus resulting in a significant decrease in transparency. ${ }^{\mathbf{3 4}}$

To further study the surface properties of these substrates, tapping-mode atomic force microscopy (AFM) was adopted to measure the surface morphologies of PVA/sucrose and PVA/ gelatin films. The AFM results are presented in Fig. 4 and $\mathrm{S} 2 . \dagger$ For each film, the surface close to the glass substrate was used for AFM measurement. As can be seen, PVA films showed highly uniform small grains with the root-mean-square (RMS) value of surface roughness of $1.42 \mathrm{~nm}$. The RMS values were $1.89 \mathrm{~nm}$, $1.91 \mathrm{~nm}, 2.24 \mathrm{~nm}, 1.57 \mathrm{~nm}$ for PS10, PS2, PS1 and PS0.5, and $1.95 \mathrm{~nm}, 2.46 \mathrm{~nm}$ and $1.65 \mathrm{~nm}$ for PG10, PG2 and PG1, respectively. It can be found that for both PVA/sucrose and PVA/gelatin substrates, the surface roughness of samples

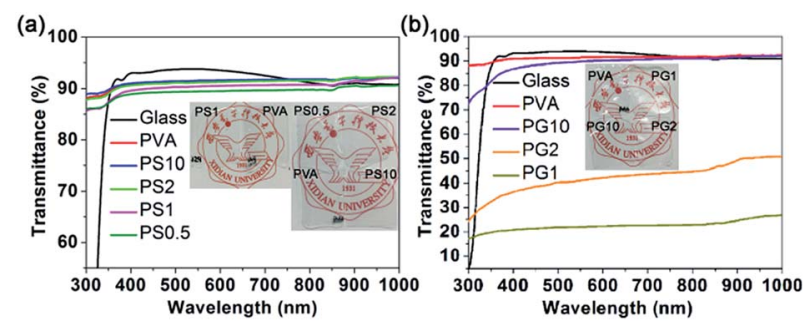

Fig. 3 Transmittance spectra of the free-standing PVA/sucrose (a) and PVA/gelatin (b) matrix substrates. Inset shows the optical images of the polymer substrates. 


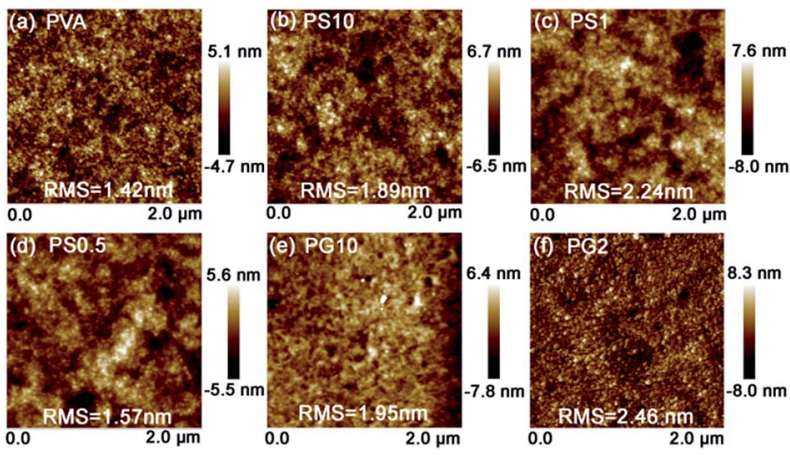

Fig. 4 Surface AFM images $(2 \times 2 \mu \mathrm{m})$ of PVA films without (a) and with different ratios of $(b-e)$ sucrose or $(f)$ gelatin.

increases with the increased filler concentrations, which is probably due to the increase in the surface undulation and the crystal size. However, the samples of substrates with the filler concentrations equal or higher than that of PVA (i.e. PS0.5 and PG1) exhibit a decreased surface roughness. The reason for RMS decrease maybe that the slack of the crystalline domains in polymers caused by high concentration of sucrose or the formation of continuous phase of the gelatin. Since the surface facing to the supporting substrates was adopted in the measurement and device fabrication, the surface of the above samples is smooth. So further surface planarization of the substrates is not needed in this study.

To fabricate transient organic solar cells on the above PVA-based substrates, a transparent conductors is required for efficient light absorption of the active layer. Here, $\mathrm{Ag}$ thin film was chosen as transparent anode due to its high conductivity, intrinsic flexibility and good transmittance so that it could be compatible with PVA substrates. In previous work we have confirmed that the optimal thickness of Ag transparent electrode is $11 \mathrm{~nm}$ (i.e. percolation threshold of thermally evaporated $\mathrm{Ag}$ film in our laboratory), which exhibits good electrical conductivity and optical transparency. ${ }^{42}$ So in this study, $11 \mathrm{~nm}$ $\mathrm{Ag}$ layer was thermally evaporated on the polymer substrates to serve as the transparent bottom electrode. The $\mathrm{MoO}_{3}$ layer was utilized as the hole-transporting layer with high work function to fabricated the planar heterojunction OSCs, PVA substrate/Ag/ $\mathrm{MoO}_{3} / \mathrm{P} 3 \mathrm{HT}: \mathrm{PC}_{61} \mathrm{BM} / \mathrm{Ca} / \mathrm{Ag}$. Fig. 5a and c illustrate the schematic device structure and energy level diagram of each component in the transient OSCs. The fabrication procedure of
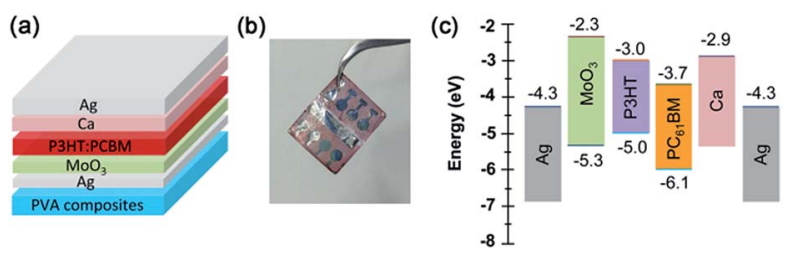

Fig. 5 (a) Schematic device structure of the transient OSCs prepared on PVA-based substrates (PVA/Ag/ $\mathrm{MoO}_{3} / \mathrm{P} 3 \mathrm{HT}: \mathrm{PC}_{61} \mathrm{BM} / \mathrm{Ca} / \mathrm{Ag}$ ). (b) Optical image of the fabricated OSCs on PVA substrate. (c) Energy level diagram of materials in the OSCs. the PVA-based substrates and the transient OSCs are presented in Fig. S3. $\dagger$ Glass substrates were employed as supporting substrate during the OSCs fabrication. A prepared solar cell on PVA substrate is shown in Fig. 5b. The specular reflection of Ag electrode reveals the uniform active layer and high surface smoothness of the PVA substrate.

The current density-voltage $(J-V)$ characteristics of OSCs on PVA/sucrose and PVA/gelatin substrates are depicted in Fig. 6. The photovoltaic performance parameters are summarized in Table 1 . The $J-V$ curves for the reference OSCs on glass substrate are also shown for comparison. The substrate properties significantly affect the photovoltaic performance of OSCs. As can be seen, a PCE of $1.83 \%$ is achieved for the OSCs fabricated on PVA substrates, with a short-circuit current density $\left(J_{\mathrm{sc}}\right)$ of $5.21 \mathrm{~mA} \mathrm{~cm}{ }^{-2}$, open-circuit voltage $\left(V_{\mathrm{oc}}\right)$ of $0.62 \mathrm{~V}$, and fill factor (FF) of $56.7 \%$, which is relatively lower than that (PCE of 2.37\%) of the reference devices on glass substrates. The decrease of the device performance mainly lies in $J_{\mathrm{sc}}$ and $\mathrm{FF}$, and can be ascribed from the lower transparency and increased surface roughness of PVA compared to the glass substrate. For OSCs on sucrose-based substrates, the $J_{\mathrm{sc}}$ of OSC on PS2 substrate is 5.72 $\mathrm{mA} \mathrm{cm}{ }^{-2}$, which is higher than that $\left(5.51 \mathrm{~mA} \mathrm{~cm}{ }^{-2}\right)$ of the devices on PS10 substrate. However, increasing the sucrose concentrations to equal or higher than that of PVA does not further increase the $J_{\mathrm{sc}}$. The $J_{\mathrm{sc}}$ value for OSCs fabricated on PS1 and PSO.5 substrates is $4.4 \mathrm{~mA} \mathrm{~cm}{ }^{-2}$ and $5.29 \mathrm{~mA} \mathrm{~cm}^{-2}$, respectively. It has been found that the surface morphology and property of metal electrode is closely related to the substrate, and different substrates will result in different surface morphology of Ag electrodes. ${ }^{42,43}$ As we discussed above, the RMS increases from $1.89 \mathrm{~nm}$ for PS10 to $1.91 \mathrm{~nm}$ for PS2. The appropriate increase in the surface roughness of substrates may improve the contact between the bottom $\mathrm{Ag}$ electrode and $\mathrm{MoO}_{3}$ buffer layer, thus leading to an increased $J_{\text {sc }}$ in PS2-based OSCs\%. But meanwhile, the Ag surface defects increase with the increasing roughness, which further induce interface
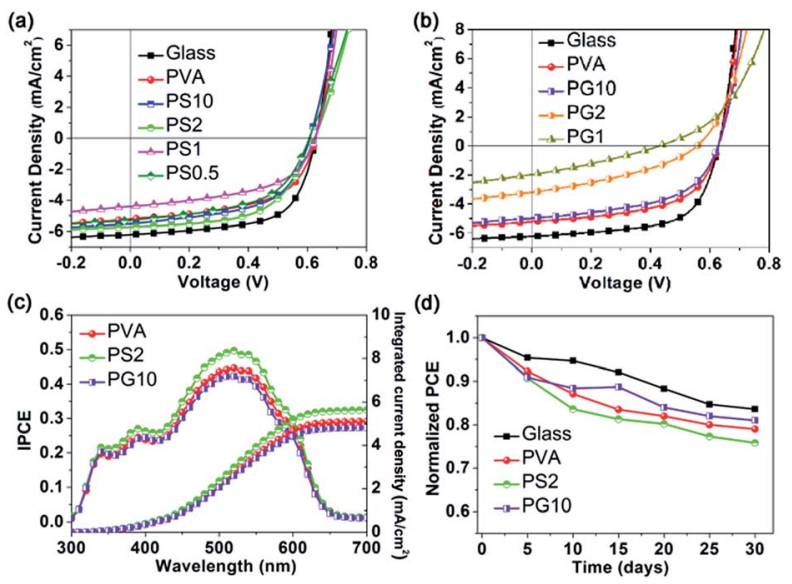

Fig. 6 J-V characteristics of the transient OSCs fabricated on (a) PVA/ sucrose and (b) PVA/gelatin substrates under 100 AM 1.5G illumination of $100 \mathrm{~mW} \mathrm{~cm}^{-2}$ (c) IPCE spectra of the OSCs fabricated on PVA, PS2 and PG10 substrates. (d) The stability of the transient OSCs prepared on glass, PVA, PS2 and PG10 substrates. 
Table 1 Photovoltaic parameters of OSCs fabricated on glass substrate and PVA composite substrates with varying compositions

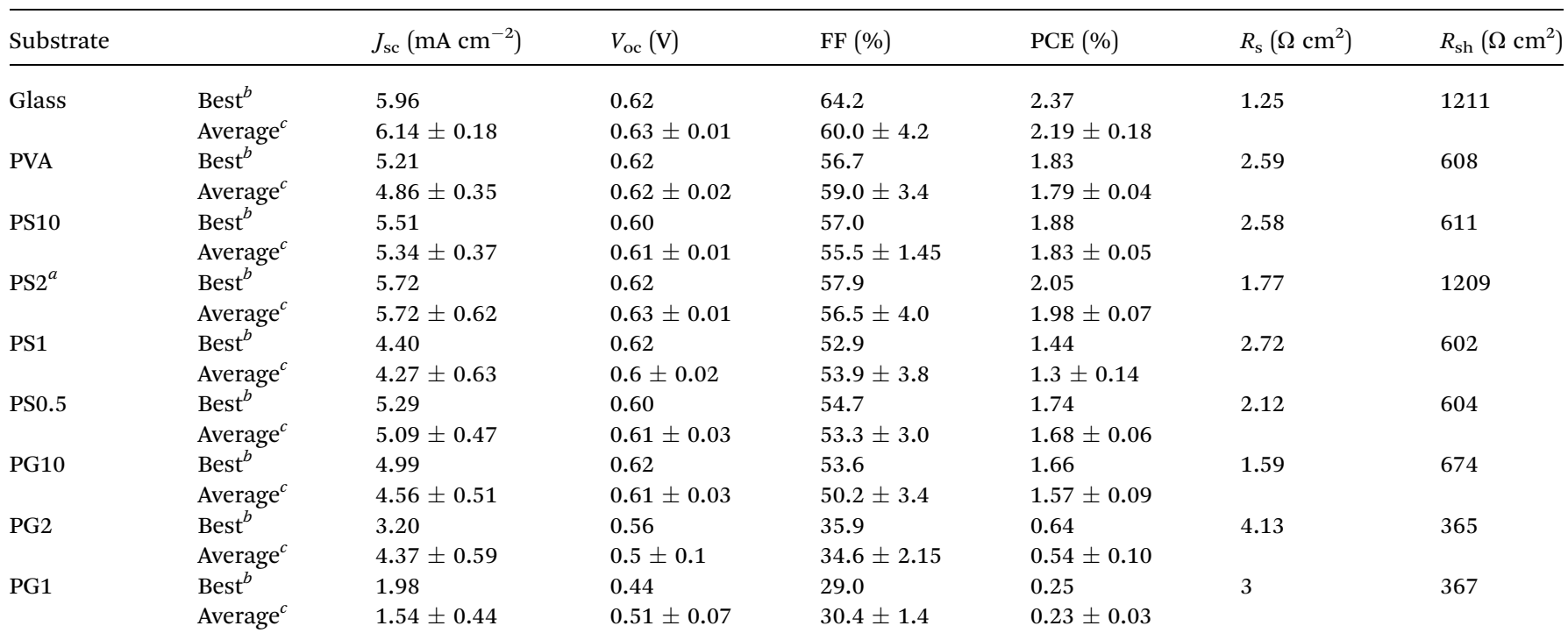

${ }^{a}$ The best device was achieved on PS2 substrate with PVA-to-sucrose ratio of $2: 1 .{ }^{b}$ Maximum values of two batches of 8 solar cells. ${ }^{c}$ The average results were derived from two batches counting 8 solar cells in total.

carrier's recombination, thus, decreased $J_{\mathrm{sc}}$ in PS1-based devices. This is also in agreement with the variation in series resistance $\left(R_{\mathrm{S}}\right) . R_{\mathrm{S}}$ decreases from $2.59 \Omega \mathrm{cm}^{2}$ (for OSCs on pure PVA substrates) to $1.77 \Omega \mathrm{cm}^{2}$ for devices on PS2 substrates. Additionally, the PS2 substrates show excellent transparency in the visible and near infrared spectra range (Fig. 3a), which is comparable to the glass substrate and contributes to the high $J_{\mathrm{sc}}$ value. Apart from $J_{\mathrm{sc}}$, the sucrose content in the substrates also affects other photovoltaic parameters. FF of the OSCs on PS2 substrate is $57.9 \%$, which is higher than that of the devices on PVA or other sucrose-based substrates. The changes in the FF are related to the increase of the shunt resistance (Table 1). Therefore, the device prepared on PS2 substrate delivers the best performance among all OSCs on sucrose-based substrates, with a $J_{\text {sc }}$ of $5.72 \mathrm{~mA} \mathrm{~cm} \mathrm{~cm}^{-2}, V_{\mathrm{oc}}$ of $0.62 \mathrm{~V}, \mathrm{FF}$ of $57.9 \%$, and a remarkable PCE of $2.05 \%$, comparable to the $2.37 \%$ PCE of the reference device based on glass substrates with a $J_{\mathrm{sc}}$ of $5.92 \mathrm{~mA} \mathrm{~cm}^{-2}, V_{\text {oc }}$ of $0.62 \mathrm{~V}, \mathrm{FF}$ of $64.2 \%$. Although this result is modest compared to the reference devices on glass substrates, it reveals a significant improvement over previous reported P3HT: $\mathrm{PC}_{61} \mathrm{BM}$ based solar cells on paper-like or cellulose nanocrystal (CNC) substrates, and can be further improved through optimization of the interface between the substrate and Ag electrode..$^{30-32,44}$

As for OSCs on gelatin-based substrates, the $J_{\mathrm{sc}}$ is strikingly decreased from $4.99 \mathrm{~mA} \mathrm{~cm}^{-2}$ to $1.98 \mathrm{~mA} \mathrm{~cm}^{-2}$ with the PVA-togelatin ratios decreasing from $10: 1$ to $1: 1$ (Fig. 6b). The decreased $J_{\mathrm{sc}}$ ascribes to the significant decrease in the transparency of the substrates. Therefore, the devices on PG2 and PG1 substrates exhibit low efficiency of $0.64 \%$ and $0.25 \%$, respectively. The best-performing device fabricated on gelatinbased substrates is achieved on PG10 substrates, which shows a $V_{\text {oc }}$ of $0.62 \mathrm{~V}, J_{\mathrm{sc}}$ of $4.99 \mathrm{~mA} \mathrm{~cm}{ }^{-2}, \mathrm{FF}$ of $53.6 \%$, and an overall PCE of $1.66 \%$.
The incident photo-to-current efficiency (IPCE) curves of these OSCs on PVA-based substrates are presented in Fig. $6 \mathrm{c}$ and S4. $\uparrow$ The filler type and PVA-to-filler ratios could affect the photo-to-electricity conversion efficiency. The devices on PS2 substrate shows IPCE value up to $50 \%$ within the spectra range of 340-650 nm, which is much higher than that of devices on PVA (45\%) and PG10 (43\%) substrates. The integrated $J_{\text {sc }}$ values are 5.69, 5.16 and $4.87 \mathrm{~mA} \mathrm{~cm}^{-2}$ for devices on PS2, PVA and PG10 substrates, respectively, which coincide well with the measure $J_{\mathrm{sc}}$ within the errors. In addition, the device stability of the above solar cells was also investigated periodically for the un-encapsulated cells. These cells were stored in nitrogen-filled glove box and measured in air ambient conditions. The normalized PCEs as a function of the storage time are displayed in Fig. 6d. As can be seen, the PCE of the reference solar cell on glass substrate exhibits a good stability, which maintains $84 \%$ of the initial efficiency after 30 days. In contrast, the PCEs of the cells on PVA-based substrates exhibit a relatively fast degradation, with about $76-81 \%$ remaining of the original PCEs. The energy loss is mainly due to the oxidation of Ca layer and penetration of water or oxygen into the active layer, and also the intrinsic flexibility of the substrates. Thus better stability can be expected for our encapsulated solar cells by avoiding mechanical deterioration and oxidation.

To study the transiency behaviours of the OSCs on PVAbased substrates, we submersed the cells in DI water at room temperature, and recorded the dissolution time and transient behaviour of the devices (Fig. 7 and $55 \dagger$ ). When the transient OSCs are immersed into water, the transiency behaviour undergoes four stages: (1) the generation of expansion force as a result of swelling of the polymer substrates when triggered by water, (2) integrity of the device arrays prevents expansion of polymer chains in substrate, which are in contact with the device, (3) the device arrays start to disintegrate as the 

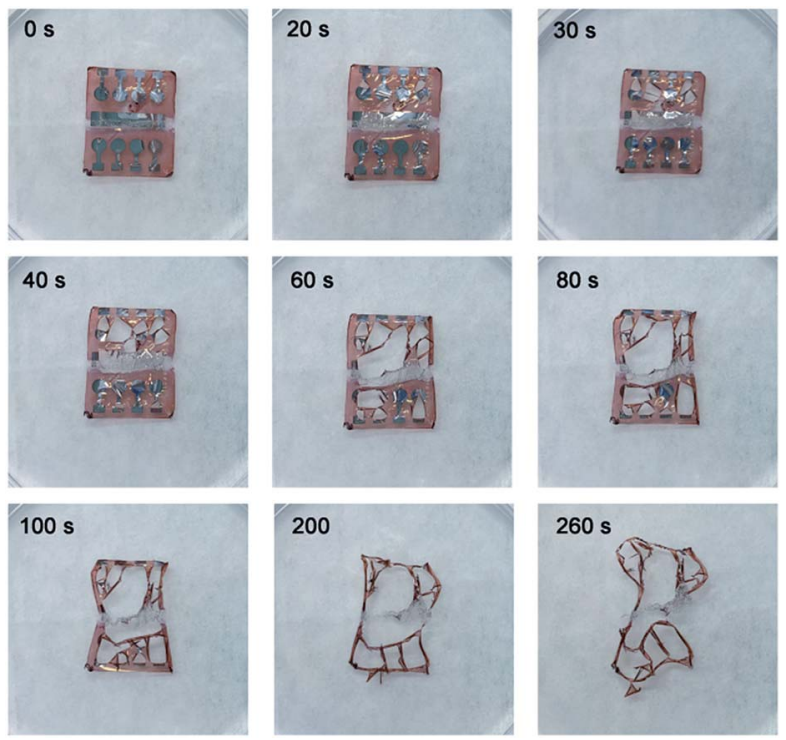

Fig. 7 Optical pictures illustrating time sequence of the dissolution of transient OSCs on PVA substrate in DI water.

expansion of substrates achieved a critical point, and (4) the complete dissolution of the substrates and breaking of device arrays to individual particles. Since the physical breaking of the devices is essentially caused by the force generated by swelling of the substrates, the disintegration time of the transient solar cells directly depends on the dissolution behavior of the substrates (i.e. the chemical and physical interactions between/ within substrate, water and the components of OSCs). Fig. 7 shows the transiency test of the cells on PVA substrate in DI water. Once immersed in water, the PVA substrate swells and dissolves quickly until it cannot hold the integrity of the devices, leading to the disconnection of the cells only in $20 \mathrm{~s}$ exposure. Upon further dissolution, the photoactive layer of the devices wrapped, with the bottom Ag electrode disintegrating to individual particles after $40 \mathrm{~s}$ of exposure. A video available in ESI $\dagger$ clearly shows the transiency process where the solar cells on PVA substrate can easily disintegrate under the constant dripping of water. By applying small amount of sucrose to PVA, the PS2 substrate displays much faster dissolution and expansion in first couple of seconds compared with PVA substrate. As shown in Fig. S5a, $\dagger$ the device arrays could not resist the strong expansion force and disintegrated in only $10 \mathrm{~s}$ of exposure to DI water, exhibiting very fast transiency. The substrate with PVA/ gelatin ratio of $2: 1$ shows the largest expansion and slowest dissolution among other two samples. The photoactive layer and electrodes on PG2 substrates were significantly wrapped and disconnected into individual particles after $80 \mathrm{~s}$ of immersion in water, with the particles being distributed over the whole substrate (Fig. 5Sb $\dagger$ ). However, the devices do not break even after an hour of exposure. There results clearly indicated that the transiency of such transient OSCs can be controlled and programmed through the dissolution and swelling properties of the substrates, which can be further manipulated by appropriate choice of the polymer compositions.

\section{Conclusions}

In summary, for the first time, we reported physically transient organic solar cells fabricated on free-standing PVA-based substrates with the configuration of PVA composite/Ag/ $/ \mathrm{MoO}_{3} /$ $\mathrm{P} 3 \mathrm{HT}: \mathrm{PC}_{61} \mathrm{BM} / \mathrm{Ca} / \mathrm{Ag}$. We found that the photovoltaic performance and transiency behavior of the transient OSCs is mainly dependent on the dissolution and optical transmittance characteristics of PVA substrates, which can further be programmed by means of addition of sucrose or gelatin at different ratios. By applying PS2 thin film as a substrate, a PCE up to $2.05 \%$ was obtained, which is much higher than other reported P3HT:PC ${ }_{61} \mathrm{BM}$ based solar cells on paper-like or CNC substrates. Once triggered by DI water, the whole device disintegrated into an unrecognized extent only after $10 \mathrm{~s}$ of exposure, relatively shorter than that of devices on PVA substrates $(20 \mathrm{~s})$. Compared with these two samples, the devices on PG2 substrates manifested the slowest dissolution, which did not disconnect even after an hour of immersion in water, but in the form of individual particles. The demonstration of physically transient OSCs on PVA-based substrates opens a door for future development of transient organic photovoltaic technology to fulfill the energy demands of transient applications such as remote environmental monitoring.

\section{Experimental}

\section{Materials and chemicals}

All materials were used as received without further purification. P3HT was bought from American Dye Source Inc. $\mathrm{PC}_{61} \mathrm{BM}$ was purchased from Nano-C Inc. Other materials, including $\mathrm{MoO}_{3}$, 1,2-dichlorobenzene (anhydrous, 99\% purity), PVA ( $M_{\mathrm{w}}$ : $61000 \mathrm{~g} \mathrm{~mol}^{-1}, 98-98.8 \mathrm{~mol} \%$ hydrolysis), sucrose (99\% purity), gelatin (from porcine skin) were obtained from Sigma-Aldrich.

\section{Preparation of the polymer substrates}

Thin films of PVA composites were prepared according to the literature. ${ }^{24}$ For the polymeric matrix, 5 wt $\%$ PVA solution was prepared by dissolving $1 \mathrm{~g}$ PVA in $20 \mathrm{~mL}$ deionized water, into which $10 \mu \mathrm{L} \mathrm{HCl}$ aqueous solution $(1 \mathrm{M})$ was added to accelerate the dissolution. To synthesize PVA/gelatin and PVA/sucrose blends, a certain amount of fillers (gelatin or sucrose) was added into the base PVA solution. The mixture was stirred at $120{ }^{\circ} \mathrm{C}$ for $3 \mathrm{~h}$ until it completely dissolved, then the obtained solution was cooled to room temperature and stirred overnight. All polymer films were prepared via drop casting method, and glass substrates were used as supporting substrate. After drying at ambient conditions for $48 \mathrm{~h}$, thin films were carefully peeled off from the supporting substrate for further characterization.

\section{Characterization of polymer films}

The transmission spectra were measured on a Perkin-Elmer Lambda 950 spectrophotometer. Attenuated total reflectanceFourier transform infrared (ATR-FTIR) spectroscopy was employed to investigate the molecular structures of these PVA composites, thus understanding the chemical interactions between PVA and the fillers. The surface morphology and 
roughness of the polymer films were measured by a Veeco NanoScope V Multi-Mode Atomic Force Microscopy operated in trapping mode (TM-AFM, Dimension Icon, Bruker).

\section{Transiency test}

The dissolution experiments were carried out via recording the mass ratio of the polymer films before and after submersion to deionized (DI) water for a certain time. The film specimens with $1 \times 3.5 \mathrm{~cm}^{2}$ were cut from each of the PVA composite films and separately placed in a closed container made by copper mesh. Following that, the mesh containers with samples in were immersed into DI water for $20,200,400,600$ and $1200 \mathrm{~s}$, respectively, and then dried at ambient conditions for $24 \mathrm{~h}$. The mass of each container and sample was recorded before and after immersing in DI water. It is notated that all experiments were taken three times to obtain the average values.

\section{Device fabrication and characterization}

For devices based on PVA composite substrates, the polymer films were firstly peeled off from the glass without further planarization, then placed onto the supporting substrate with the side close to the glass facing up. Then these samples were transferred into a metal evaporation chamber, wherein a $11 \mathrm{~nm}$ Ag film were thermally deposited onto the substrates at a vacuum pressure below $5.0 \times 10^{-4} \mathrm{~Pa}$. Following that, a $10 \mathrm{~nm}$ $\mathrm{MoO}_{3}$ layer was thermally deposited onto Ag-coated polymer substrate to act as the hole transport layer. To make a uniform active layer, a precursor solution of $\mathrm{P} 3 \mathrm{HT}$ and $\mathrm{PC}_{61} \mathrm{BM}$ blend with a weight ratio of $1: 0.8$ in 1,2-dichlorobenzene $(20 \mathrm{mg}$ $\mathrm{mL}^{-1}$ ) was stirred at room temperature for $2 \mathrm{~h}$ in a nitrogenfilled glovebox before use. Then the solution was spin coated onto the $\mathrm{MoO}_{3}$ layer at $1000 \mathrm{rpm}$ for $60 \mathrm{~s}$, followed by annealing at $90{ }^{\circ} \mathrm{C}$ for $10 \mathrm{~min}$. Finally, a Ca layer $(10 \mathrm{~nm})$ and an $\mathrm{Ag}$ layer $(70 \mathrm{~nm})$ were deposited on top of the active layer as the cathode through a shadow mask to create a device area of $0.125 \mathrm{~cm}^{2}$. For the reference devices based on glass substrate, the substrates were cleaned sequentially with detergent (Decon 90), deionized water, acetone and isopropanol in an ultrasonic bath for $15 \mathrm{~min}$ each, and then dried with a $\mathrm{N}_{2}$ stream for device fabrication. The reference devices were prepared as the same process of PVA-based OSCs.

All of the measurements were carried out under ambient atmosphere at room temperature without encapsulation. The $J-V$ characteristics were recorded using a Keithley 2400 source meter unit under AM 1.5G illumination (XES-70S1, San-Ei Electric) with an intensity of $100 \mathrm{~mW} \mathrm{~cm}{ }^{-2}$. The system was accurately adjusted using a standard silicon solar cell calibrated by the National Renewable Energy Laboratory (NREL) before device testing. The incident photo-to-current conversion efficiency (IPCE) of the transient devices was recorded by the solar cell quantum efficiency measurement system (SCS10-X150; Zolix instrument. Co. Ltd).

\section{Conflicts of interest}

There are no conflicts to declare.

\section{Acknowledgements}

We thank the National Natural Science Foundation of China (Grant No. 61704128, 61574107, 11604250), Class General Financial Grant from the China Postdoctoral Science Foundation (Grant No. 2016M602771), Opening Project of Key Laboratory of Microelectronic Devices \& Integrated Technology (Grant No. 2016M602771), and the Key Research and Development Plan Project of Shandong Province (grant no. 2015GGX104012) for financial support.

\section{Notes and references}

1 S.-W. Hwang, H. Tao, D.-H. Kim, H. Cheng, J.-K. Song, E. Rill, M. A. Brenckle, B. Panilaitis, S. M. Won, Y.-S. Kim, Y. M. Song, K. J. Yu, A. Ameen, R. Li, Y. Su, M. Yang, D. L. Kaplan, M. R. Zakin, M. J. Slepian, Y. Huang, F. G. Omenetto and J. A. Rogers, Science, 2012, 337, 16401644.

2 H. Wang, B. Zhu, X. Ma, Y. Hao and X. Chen, Small, 2016, 12, 2715-2719.

3 C. Dagdeviren, S.-W. Hwang, Y. Su, S. Kim, H. Cheng, O. Gur, R. Haney, F. G. Omenetto, Y. Huang and J. A. Rogers, Small, 2013, 9, 3398-3404.

4 S.-W. Hwang, X. Huang, J.-H. Seo, J.-K. Song, S. Kim, S. HageAli, H.-J. Chung, H. Tao, F. G. Omenetto, Z. Ma and J. A. Rogers, Adv. Mater., 2013, 25, 3526-3531.

5 H. Tao, S.-W. Hwang, B. Marelli, B. An, J. E. Moreau, M. Yang, M. A. Brenckle, S. Kim, D. L. Kaplan, J. A. Rogers and F. G. Omenetto, Proc. Natl. Acad. Sci. U. S. A., 2014, 111, 17385-17389.

6 S.-K. Kang, S.-W. Hwang, S. Yu, J.-H. Seo, E. A. Corbin, J. Shin, D. S. Wie, R. Bashir, Z. Ma and J. A. Roges, Adv. Funct. Mater., 2015, 25, 1789-1797.

7 L. Yin, X. Huang, H. Xu, Y. Zhang, J. Lam, J. Cheng and J. A. Rogers, Adv. Mater., 2014, 26, 3879-3884.

8 M. Irimia-Vladu, Chem. Soc. Rev., 2014, 43, 588-610.

9 M. A. Brenckle, H. Cheng, S. Hwang, H. Tao, M. Paquette, D. L. Kaplan, J. A. Rogers, Y. Huang and F. G. Omenetto, ACS Appl. Mater. Interfaces, 2015, 7, 19870-19875.

10 S.-W. Hwang, G. Park, C. Edwards, E. A. Corbin, S.-K. Kang, H. Cheng, J.-K. Song, J.-H. Kim, S. Yu, J. E. Lee, J. Kim, C. Yee, B. Bhaduri, Y. Su, F. G. Omennetto, Y. Huang, R. Bashir, L. Goddard, G. Popescu, K.-M. Lee and J. A. Rogers, ACS Nano, 2014, 8, 5843-5851.

11 S.-W. Hwang, J.-K. Song, X. Huang, H. Cheng, S.-K. Kang, B. H. Kim, J.-H. Kim, S. Yu, Y. Huang and J. A. Rogers, Adv. Mater., 2014, 26, 3905-3911.

12 S.-K. Kang, G. Park, K. Kim, S.-W. Hwang, H. Cheng, J. Shin, S. Chung, M. Kim, L. Yin, J. C. Lee, K.-M. Lee and J. A. Rogers, ACS Appl. Mater. Interfaces, 2015, 7, 9297-9305.

13 S. H. Jin, S.-K. Kang, I.-T. Cho, S. Y. Han, H. U. Chung, D. J. Lee, J. Shin, G. W. Baek, T.-I. Kim, J.-H. Lee and J. A. Rogers, ACS Appl. Mater. Interfaces, 2015, 7, 8268-8274.

14 S.-W. Hwang, S.-K. Kang, X. Huang, M. A. Brenckle, F. G. Omennetto and J. A. Rogers, Adv. Mater., 2015, 27, 47-52. 
15 S.-W. Hwang, D.-H. Kim, H. Tao, T.-I. Kim, S. Kim, K. J. Yu, B. Panilaitis, J.-W. Jeong, J.-K. Song, F. G. Omennetto and J. A. Rogers, Adv. Funct. Mater., 2013, 23, 4087-4093.

16 S.-K. Kang, S.-W. Hwang, H. Cheng, S. Yu, B. H. Kim, J.-H. Kim, Y. Huang and J. A. Rogers, Adv. Funct. Mater., 2014, 24, 4427-4434.

17 L. Yin, H. Cheng, S. Mao, R. Haasch, Y. Liu, X. Xie, S.-W. Hwang, H. Jain, S.-K. Kang, Y. Su, R. Li, Y. Huang and J. A. Rogers, Adv. Funct. Mater., 2014, 24, 645-658.

18 Y. F. Zheng, X. N. Gu and F. Witte, Mater. Sci. Eng. R. Rep., 2014, 77, 1-34.

19 D. Son, J. Lee, D. J. Lee, R. Ghaffari, S. Yun, S. J. Kim, J. E. Lee, H. R. Cho, S. Yoon, S. Yang, S. Lee, S. Qiao, S. Ling, S. Shin, J.-K. Song, J. Kim, T. Kim, H. Lee, J. Kim, M. Soh, N. Lee, C. S. Hwang, S. Nam, N. Lu, T. Hyeon, S. H. Choi and D.-H. Kim, ACS Nano, 2015, 9, 5937-5946.

20 S.-W. Hwang, G. Park, H. Cheng, J.-K. Song, S.-K. Kang, L. Yin, J.-H. Kim, F. G. Omennetto, Y. Huang, K.-M. Lee and J. A. Rogers, Adv. Mater., 2014, 26, 1992-2000.

21 H. Zhu, Z. Xiao, D. Liu, Y. Li, N. J. Weadock, Z. Fang, J. Huang and L. Hu, Energy Environ. Sci., 2013, 6, 2105-2111.

22 A. H. Najafabadi, A. Tamayol, N. Annabi, M. Ochoa, P. Mostafalu, M. Akbari, M. Nikkhah, R. Rahimi, M. R. Dokmeci, S. Sonkusale, B. Ziaie and A. Khademhosseini, Adv. Mater., 2014, 26, 5823-5830.

23 K. Nagashima, H. Koga, U. Celano, F. Zhuge, M. Kanai, S. Rahong, G. Meng, Y. He, J. D. Boeck, M. Jurczak, W. Vandervorst, T. Kitaoka, M. Nogi and T. Yanagida, Sci. Rep., 2014, 4, 5532.

24 H. Acar, S. Çlnar, M. Thunga, M. R. Kessler, N. Hashemi and R. Montazami, Adv. Funct. Mater., 2014, 24, 4135-4143.

25 D. N. Rockwood, R. C. Preda, T. Yücel, X. Wang, M. Lovett and D. L. Kaplan, Nat. Protoc., 2011, 6, 1612-1631.

26 K. K. Fu, Z. Wang, J. Dai, M. Carter and L. Hu, Chem. Mater., 2016, 11, 3527-3539.

27 Y. Zhou, C. Fuentes-Hernandez, T. M. Khan, J.-C. Liu, J. Hsu, J. W. Shim, A. Dindar, J. P. Youngblood, R. J. Moon and B. Kippelen, Sci. Rep., 2013, 3, 1536.

28 Z. Liu, K. Fu, Z. Wang, Y. Zhu, J. Wan, Y. Yao, J. Dai, M. Kim, L. Swafford, C. Wang and L. Hu, Inorg. Chem. Front., 2016, 3, 681-688.
29 K. Fu, Z. Wang, C. Yan, Z. Liu, Y. Yao, J. Dai, E. Hitz, Y. Wang, W. Luo, Y. Chen, M. Kim and L. Hu, Adv. Energy Mater., 2016, 6, 1502496.

30 T.-S. Kim, S.-I. Na, S.-S. Kim, B.-K. Yu, J.-S. Yeo and D.-Y. Kim, Phys. Status Solidi RRL, 2012, 6, 13-15.

31 L. Hu, G. Zheng, J. Yao, N. Liu, B. Weil, M. Eskilsson, E. Karabulut, Z. Ruan, S. Fan, J. T. Bloking, M. D. McGehee, L. Wågberg and Y. Cui, Energy Environ. Sci., 2013, 6, 513-518.

32 F. Wang, Z. Chen, L. Xiao, B. Qu and Q. Gong, Sol. Energy Mater. Sol. Cells, 2010, 94, 1270-1274.

33 L. Ye, W. Zhao, S. Li, S. Mukherjee, J. H. Carpenter, O. Awartani, X. Jiao, J. Hou and H. Ade, Adv. Energy Mater., 2017, 7, 1602000.

34 H. Acar, S. Banerjee, H. Shi, R. Jamshidi, N. Hashemi, M. W. Cho and R. Montazami, Materials, 2016, 9, 321.

35 C. J. Bettinger and Z. Bao, Adv. Mater., 2010, 22, 651-655.

36 S. H. Jin, J. Shin, I.-T. Cho, S. Y. Han, D. J. Lee, C. H. Lee, J.-H. Lee and J. A. Rogers, Appl. Phys. Lett., 2014, 105, 093506.

37 S. Çınar, R. Jamshidi, Y. Chen, N. Hashemi and R. Montazami, J. Polym. Sci., Part B: Polym. Phys., 2016, 54, 517-524.

38 S. M. Pawde and K. Deshmukh, J. Appl. Polym. Sci., 2008, 109, 3431-3437.

39 A. Bigi, S. Panzavolta and K. Rubini, Biomaterials, 2004, 25, 5675-5680.

40 G. I. Andrade, E. F. Barbosa-Stancioli, A. A. Piscitelli Mansur, W. L. Vasconcelos and H. S. Mansur, J. Mater. Sci., 2008, 43, 450-463.

41 C. Xiao, H. Liu, Y. Lu and L. Zhang, J. Macromol. Sci., Part A: Pure Appl.Chem., 2001, 38, 317-328.

42 Z. Wang, C. Zhang, R. Gao, D. Chen, S. Tang, J. Zhang, D. Wang, X. Lu and Y. Hao, Sol. Energy Mater. Sol. Cells, 2014, 127, 193-200.

43 Z. Wang, C. Zhang, D. Chen, S. Tang, J. Zhang, Y. Wang, G. Han, S. Xu and Y. Hao, IEEE Photonics J., 2015, 7, 8400109.

44 A. Hübler, B. Trnovec, T. Zillger, M. Ali, N. Wetzold, M. Mingebach, A. Wagenpfahl, C. Deibel and V. Dyakonov, Adv. Energy Mater., 2011, 1, 1018-1022. 